PROCEEDINGS OF THE

AMERICAN MATHEMATICAL SOCIETY

Volume 127, Number 2, February 1999, Pages 609-613

S 0002-9939(99)04457-3

\title{
SMOOTHLY EMBEDDED SPHERES IN SYMPLECTIC 4-MANIFOLDS
}

\author{
TIAN-JUN LI
}

(Communicated by Ronald A. Fintushel)

\begin{abstract}
We characterize rational or ruled surfaces among all symplectic 4-manifolds by the existence of certain smoothly embedded spheres.
\end{abstract}

\section{INTRODUCTION}

In 1989, McDuff [M1] characterized rational or ruled surfaces among all symplectic 4-manifolds by the existence of any symplectically embedded sphere with nonnegative intersection.

In this note, using Taubes's remarkable results on Seiberg-Witten invariants of symplectic 4-manifolds, we show that rational or ruled surfaces can be further characterized among all symplectic 4-manifolds by the existence of certain smoothly embedded spheres. More precisely, we have

Theorem 1. Let $M$ be a symplectic 4-manifold with symplectic canonical class $K$. If $F \in H_{2}(M ; \mathbf{Z})$ is represented by a smoothly embedded sphere with self-intersection -1 and $K(F) \neq \pm 1$, then $M$ is rational or ruled, and after an orientation-preserving diffeomorphism, up to sign, $F$ is $\mathbf{Z}$-homologous to a symplectic - 1 curve with respect to the new symplectic form.

Corollary 2. Let $M$ be a symplectic 4-manifold. If there is a smoothly embedded sphere with nonnegative self-intersection and of infinite order in $H_{2}(M ; \mathbf{Z})$, then $M$ is rational or ruled, and there is a symplectically embedded sphere with nonnegative self-intersection.

Another result we obtain is

Corollary 3. Let $M$ be a symplectic 4-manifold, which is not rational nor ruled. Then every smoothly embedded - 1 sphere is $\mathbf{Z}$-homologous to a symplectic -1 curve up to sign. If $M$ is the blow up of a minimal symplectic 4 -manifold with $E_{1}, \ldots, E_{l}$ represented by exceptional curves, then the $E_{i}$ are the only classes represented by a smoothly embedded -1 sphere, hence any orientation-preserving diffeomorphism maps $E_{i}$ to some $\pm E_{j}$.

Our Theorem 1, Corollary 2 and Corollary 3 are generalizations of the relevant results in $[\mathrm{B}]$ and $[\mathrm{FM}]$, where Brussee, Friedman and Morgan worked in the category of Kähler surfaces. Fintushel and Stern [FS1] already showed that the manifold

Received by the editors January 31, 1997 and, in revised form, April 4, 1997.

1991 Mathematics Subject Classification. Primary 57Rxx.

(C)1999 American Mathematical Society 
$M$ in Corollary 2 must have $b_{2}^{+}=1$. However, there are non-Kähler symplectic 4 -manifolds with $b_{2}^{+}=1$, and many simply connected examples were constructed recently by Fintushel and Stern [FS2].

Liu [Liu] gave some other very interesting characterizations of rational and ruled surfaces. McDuff [M3] proved that rational or ruled surfaces are the only symplectic 4-manifolds with possibly more than two minimal reductions. This also follows from Corollary 3.

The author thanks Prof. Edgar Brown and Prof. Shing-Tung Yau for advice and continuous encouragement. He also thanks Prof. Aiko Liu for useful discussions and Prof. Dusa McDuff for useful comments which corrected a mistake in an earlier draft.

\section{Proofs}

We will prove our results using Taubes's deep results [T1, T2] on Seiberg-Witten invariants on symplectic 4 -manifolds with $b_{2}^{+}=1$. For a detailed description of Seiberg-Witten invariants, see $[\mathrm{KM}],[\mathrm{W}],[\mathrm{B}],[\mathrm{FM}]$. We will only briefly describe some special features of Seiberg-Witten invariants of symplectic 4-manifolds with $b_{2}^{+}=1$.

On a 4-manifold with $b_{2}^{+}=1$, the second cohomology classes with positive square form a cone with two connected components. Pick one of them and call it the forward cone. Given a metric $g$, there is a unique self-dual harmonic 2-form $\omega_{g}$ for $g$ in the forward cone with $\omega_{g}^{2}=1$. For a closed self-dual 2-form $\mu$ and a $\operatorname{spin}^{c}$ structure $L$, define the discriminant $\Delta_{L}(g, \mu)=\int\left(c_{1}(L)-\mu\right) \omega_{g}$. Given the choice of the forward cone, $S W_{+}(L)$ and $S W_{-}(L)$ are defined for pairs $(g, \mu)$ with positive and negative discriminant respectively. For a symplectic 4-manifold, the symplectic form determines a natural choice of the forward cone.

In the following, we also recall some results about pseudo-holomorphic curves in symplectic 4-manifolds. Let $(M, \omega)$ be a symplectic 4-manifold and $J$ a compatible almost complex structure. Let $\phi$ be a $J$-holomorphic map from a possibly disconnected Riemann surface $\Sigma=\coprod \Sigma_{i}$ to $M$. For each component $\Sigma_{i}$, we can assign a multiplicity $m_{i}$. We require that the images $\phi\left(\Sigma_{i}\right)$ of different components be distinct and that none of them be a single point. $\phi, \Sigma$ and $\phi^{\prime}, \Sigma^{\prime}$ are called equivalent if their images and assigned multiplicities are the same. We define a $J$-holomorphic curve $(\phi, \Sigma)$ to be the equivalence class containing $\phi, \Sigma$. A -1 curve is an embedded $J$-holomorphic rational curve with self-intersection -1 .

Recall that $\mathcal{C}_{\omega}$ is the Fréchet space of smooth almost complex structures which are compatible with $\omega$. Given $J \in \mathcal{C}_{\omega}$ and $\alpha \in H_{2}(M ; \mathbf{Z})$ we use $H_{J}(\alpha)$ to denote the space of $J$-holomorphic curves where

1) $\Sigma$ is a compact Riemann surface;

2) $\phi_{*}[\Sigma]=\alpha$.

Now, let us state Taubes's remarkable theorems in the case $b_{2}^{+}=1$.

Theorem T1 ([T1]). Let $M$ be a symplectic 4-manifold with $b_{2}^{+}=1$ and symplectic canonical class $K$. Then $S W_{-}\left(K^{-1}\right)= \pm 1$.

Theorem T2 ([T2]). Let $M, K$ be as above, and let $e \in H^{2}(M ; \mathbf{Z})$. If

$$
S W_{-}\left(K^{-1}+2 e\right) \neq 0
$$

then the Poincaré dual of e can be represented by a pseudo-holomorphic curve. 
We first give a regularity result for pseudo-holomorphic curves.

Proposition 2.1. Let $M$ be a compact oriented 4-manifold with symplectic form $\omega$. There is a generic set in $\mathcal{C}_{\omega}$ whose members have the following property: First, fix a nonzero class $\alpha \in H_{2}(M ; \mathbf{Z})$. Then suppose $H_{J}(\alpha)$ contains $(\phi, \Sigma)$, and $S \subset \Sigma$ is the union of those components whose fundamental classes have $\phi_{*}$-images with negative square. Then there exists an element $(\tilde{\phi}, \widetilde{\Sigma})$, such that the image of $\widetilde{S}$ under $\tilde{\phi}$ is a disjoint union of -1 curves $\left(\left.\tilde{\phi}\right|_{\tilde{S}}\right.$ may not be an embedding, i.e. perhaps multiply covered) and they do not intersect the image of the other components of $\widetilde{\Sigma}$ under $\tilde{\phi}$.

Proof. It is well known (a proof is given in [M4]) that for generic $J \in \mathcal{C}_{\omega}$, if $\Xi$ is a connected Riemann surface with genus $g$ and $\psi$ is a $J$-holomorphic map from $\Xi$ to $M$ whose image has negative square, then $\Xi$ must be a rational curve, and $\psi(\Xi)$ is embedded with square -1 (though $\psi$ might not be an embedding).

Let $\Xi$ be a component in $S$. Suppose $\phi(\Xi)$ intersects the image $\phi(\Omega)$ of another component $\Omega$. By Ruan-Tian gluing ([RT]), we produce a new component whose image has nonnegative square and at the same time decrease the multiplicities on $\Xi$ and $\Omega$ by 1 . Thus we obtain another element in $\mathcal{H}_{J}(\alpha)$ and denote it by $\left(\phi_{1}, \Sigma_{1}\right)$. By repeating this process a finite number of times, we arrive at an element $(\tilde{\phi}, \widetilde{\Sigma}) \in \mathcal{H}_{J}(\alpha)$ satisfying the requirement in the proposition.

We can start to prove Theorem 1.

Proof of Theorem 1. Given any $F \in H_{2}(M ; \mathbf{Z})$, denote by $\check{F} \in H^{2}(M ; \mathbf{Z})$ the Poincaré dual of $F$. Recall that given a smoothly embedded -1 sphere representing a homology class $D$, there are diffeomorphisms of $M$, whose induced action $R(D)$ on $H^{2}(M ; \mathbf{Z})$ is a reflection along $\check{D}$. The argument in the first two paragraphs in the proof of Theorem A in [LL] gives the following

Lemma 2.2. Let $M$ be a symplectic 4-manifold with $b_{2}^{+}=1$, and let $D$ be represented by a smoothly embedded sphere with self-intersection -1 . Then

$$
S W_{-}\left(R(D)\left(K^{-1}+2 e\right)\right)=S W_{-}\left(K^{-1}+2 e\right) .
$$

By possibly changing $D$ to $-D$, we can assume $K^{-1}(D)=l>0$. Reflecting along $D, R(D)\left(K^{-1}\right)=K^{-1}+2 l \check{D}$. By Lemma 2.2 and Theorem T1, $l D$ is represented by a pseudo-holomorphic curve. Since $l D$ has negative self-intersection, by Proposition 2.1 , we can write $l D=k F+T$, where $F$ is represented by a -1 curve, $k>0, T$ is a pseudo-holomorphic curve (may not be connected) and $F \cdot T=0$. Intersecting with $F$, we find $k$ is divisible by $l$ and $D \cdot F$ is negative. Notice that we also have

$$
l \omega(D)=k \omega(F)+\omega(T) \geq l \omega(F)
$$

where $\omega$ is the symplectic form on $M$.

The key observation is that it must hold that $-(l-1) / 2 \leq D \cdot F \leq-1$. If this is not the case, i.e. $D \cdot F \leq-(l+1) / 2$, then reflecting along $D$, we get

$$
K^{-1}+2 \check{F} \rightarrow K^{-1}+2(\check{F}+\check{D})-2(l+1+D \cdot 2 F) \check{D} .
$$

By Lemma 2.2,

$$
S W_{-}\left(K^{-1}+2(\check{F}+\check{D})-2(l+1+D \cdot 2 F) \check{D}\right)=S W_{-}\left(K^{-1}+2 \check{F}\right)=S W_{-}\left(K^{-1}\right) .
$$

Therefore by Theorems $\mathrm{T} 1$ and $\mathrm{T} 2,(F-D)+(l+1+2 D \cdot F) D$ is represented by a pseudo-holomorphic curve. This is impossible, since $\omega(D)>\omega(F)>0$. 
If $D \cdot F=-(l-1) / 2$, then $\left(K^{-1}+2 F\right)(D)=1$. By Theorem A in [LL], $D$ can be represented by a symplectic -1 curve for the symplectic form $R(F)(\omega)$. Notice that $-F$ can also be represented by a symplectic -1 sphere for the symplectic form $R(F)(\omega)$ for the same reason. By the gluing technique of Ruan and Tian [RT] (or A.2 in [MS]), we can glue the two -1 curves representing $-F$ and $D$ to get an immersed symplectic sphere with nonnegative self-intersection. This symplectic sphere has pairing 2 with $K^{-1}+2 \check{F}$, so by the following theorem of McDuff [M3], $M$ is rational or ruled.

Theorem (McDuff). Let $M$ be a symplectic 4-manifold with symplectic canonical class $K$. If there is a nonnegatively immersed symplectic rational curve whose pairing with $K$ is less than -1 , then $M$ is rational or ruled.

If $-(l-1) / 2<D \cdot F \leq-1$, then $D \cdot\left(K^{-1}+2 F\right)$ is positive and strictly smaller than $l$. By repeating the above process, we complete the proof of Theorem 1.

Proof of Corollary 2. If the embedded sphere has self-intersection $l$, then by blowing up at $l$ points on this sphere, we get an embedded sphere with self-intersection zero and represent a class $C$. If $K(C) \neq 0$ and $K(C) \neq-2$, then blowing up once more, we get a smoothly embedded -1 sphere as in Theorem 1 , so the result follows from Theorem 1.

If $K(C)=0$ or $K(C)=-2$, after possibly changing the orientation, we can assume that the class $C$ has pairing 1 with $K^{-1}$. Blow up at a point on it, and let $E$ be the class represented by the exceptional curve. Then the class $C-E$ is represented by an embedded sphere satisfying the condition in Theorem A in [LL], therefore is also represented by a -1 curve. The two -1 curves intersect at one point, so we can glue them together to get an immersed rational curve with square zero. It is actually an embedded curve by the adjunction inequality, hence the theorem follows from McDuff's result cited in the beginning.

Proof of Corollary 3. Since $M$ is neither rational nor ruled, by Theorem 1, any embedded sphere with self-intersection -1 has pairing \pm 1 with $K$, hence it is Zhomologous to a -1 curve up to sign by Theorem A in [LL]. Let $M$ be the blow up of a minimal symplectic 4-manifold with exceptional curves representing classes $E_{1}, \ldots, E_{l}$. If $U$ is represented by an embedded -1 sphere, then $U$ or $-U$ is represented by a -1 curve. Without loss of generality, we assume that $U$ is represented by a -1 curve. If $U$ is different from any $E_{i}$, and $E_{i} \cdot S=0$, then by the positivity of intersection [M2], the -1 curve representing $U$ is disjoint from all the exceptional curves, and can be regarded as a -1 curve on $N$. But this contradicts the minimality of $N$. If $U$ is different from any $E_{i}$, but $E_{i} \cdot S \neq 0$ for some $i$, then the two -1 curves representing $U$ and $E_{i}$ have some positive intersection points. We can glue them together to get a nonnegatively immersed rational curve whose pairing with $K$ is less than 1 , hence $M$ is rational or ruled by McDuff's Theorem, again a contradiction. Therefore $U$ must be one of the $E_{i}$. The last statement follows from the obvious fact that any orientation-preserving diffeomorphism maps the set of embedded -1 spheres to itself.

\section{REFERENCES}

[B] R. Brussee, Some $C^{\infty}$-properties of Kahler surfaces, preprint.

[FS1] R. Fintushel and R. Stern, Immersed spheres in 4-manifolds and the immersed Thom conjecture, Turkish J. Math. 19 (1995), 145-157. MR 96j:57036 
[FS2] R. Fintushel and R. Stern, Knots, links, and 4-manifolds, preprint.

[FM] R. Friedman and J. Morgan, Algebraic surfaces and Seiberg-Witten invariants, J. Algebraic Geom. 6 (1997), 445-479. CMP 98:05

[KM] P. Kronheimer and T. Mrowka, The genus of embedded surfaces in the projective plane, Math. Res. Letters 1 (1994), 797-808. MR 96a:57073

[Liu] A. Liu, Some new applications of the general wall crossing formula, Math. Res. Letters 3 (1996), 569-585. MR 97k:57038

[LL] T. J. Li and A. Liu, Symplectic structures on ruled surfaces and a generalized adjunction inequality, Math. Res. Letters 2 (1995), 453-471. MR 96m:57052

[M1] D. McDuff, The structure of rational and ruled symplectic 4-manifolds, J. Amer. Math. Soc. 1 (1990), 679-710. MR 91k:58042; MR 93k:58098

[M2] D. McDuff, The local behavior of holomorphic curves in almost complex 4-manifolds, J. Diff. Geom. 34 (1991), 143-164. MR 93e:53050

[M3] D. McDuff, Immersed spheres in symplectic 4-manifolds, Ann. Inst. Fourier, Grenoble 42 (1992), 369-392. MR 93k:53030

[M4] D. McDuff, Lectures on Gromov invariants for symplectic 4-manifolds (Proc. NATO Summer School, Montreal), NATO Adv. Sci. Inst. Ser. C Math. Phys. Sci., 488, Kluwer Acad. Publ., Dordrecht, 1997, pp. 175-210. CMP 97:16

[MS] J-holomorphic curves and quantum cohomology, Univ. Lecture Series Vol. 6.

[R] Y. Ruan, Symplectic topology and complex surfaces, Geometry and Topology on Complex surfaces, ed. Mabuchi, Noguchi, Ochial, World Scientific Publications, Singapore, 1994. CMP 97:16

[RT] Y. Ruan and G. Tian, A mathematical theory of quantum cohomology, J. Diff. Geom. 42 (1995), 259-367. MR 96m:58033

[T1] C. H. Taubes, The Seiberg-Witten invariants and symplectic forms, Math. Res. Letters 1 (1994), 809-822. MR 95j:57039

[T2] C. H. Taubes, $S W \rightarrow G r:$ From the Seiberg-Witten equations to pseudo-holomorphic curves, J. Amer. Math. Soc. 9 (1996), 845-918. MR 97a:57033

[W] E. Witten, Monopoles and four manifolds, Math. Res. Letters 1 (1994), 769-796. MR 96d:57035

Department of Mathematics, Yale University, New Haven, Connecticut 06520

E-mail address: tli@math.yale.edu 